\title{
Minireview The role of dendritic cell precursors in tumour vasculogenesis
}

\author{
G Coukos*, $^{*}$, F Benencia', RJ Buckanovich ${ }^{2}$ and JR Conejo-Garcia ${ }^{2}$ \\ 'Abramson Family Cancer Research Institute, University of Pennsylvania, BRBIIIII, 42I Curie Blvd, Philadelphia, PA 19I04, USA; ${ }^{2}$ Center for Research on \\ Reproduction and Women's Health, University of Pennsylvania, 1315 Biomedical Research Building IIIII, 42I Curie Blvd, Philadelphia, PA 19I04, USA
}

In this review, we discuss the recent identification in vivo of a population of $\mathrm{CDI} / \mathrm{c}^{+}$cells exhibiting simultaneous expression of both endothelial and dendritic cell markers, termed vascular leukocytes (VLCs). VLCs are highly represented in human ovarian carcinomas and, depending on the milieu, can assemble into functional blood vessels or act as antigen-presenting cells. The identification of dendritic cell precursors as bipotent cells has important implications for the physiopathology and therapy of tumours. VLCs emerge as a novel therapeutic target against tumour vascularisation.

British Journal of Cancer (2005) 92, I | 82- | | 87. doi:I0.1038/sj.bjc.6602476 www.bjcancer.com

Published online 22 March 2005

(c) 2005 Cancer Research UK

Keywords: dendritic cells; angiogenesis; vascular endothelial growth factor A

Tumours require blood supply for expansive growth. Even p53null tumour cells, which have a reduced rate of apoptosis, die beyond an oxygen diffusion limit in the range of $150 \mu \mathrm{m}$ (Folkman, 2002). Therefore, growing tumours require the continual formation of neovasculature around which tumour cells will proliferate. These vessels are quite different from vessels of normal tissues, both at the morphological and molecular levels (Asahara et al, 1997; Ruoslahti, 2002). Until lately, angiogenesis, or sprouting of endothelial cells from existing vessels, was the only accepted mechanism of tumour vascularisation. More recent studies indicated that vasculogenesis, or recruitment of endothelial progenitors (EPCs) that differentiate into endothelial cells, plays an important role in the formation of tumour neovessels (Reyes et al, 2002). The origin of this endothelium may be either bone marrow-derived EPCs or local EPCs rooted within organs or vascular parenchyma. Initially, the only source of EPCs was thought to be a scarce haematopoietic stem-cell containing CD34 ${ }^{+}$ population (Rafii, 2000). However, the relative contribution of bone-marrow-derived EPCs to adult neovascularisation has been lately underscored, ranging from very little to $>50 \%$. Still, Asahara et al (1997) and Kalka et al (2000) described long before a much more frequent population of EPCs expanded in vitro from human peripheral blood. In animal models of ischaemia, these EPCs were also incorporated into sites of active angiogenesis, suggesting that a different population of EPCs may significantly contribute to physiological or pathological vascularisation.

\section{ENDOTHELIAL-LIKE DIFFERENTIATION OF DENDRITIC CELLS (DCS) IN VITRO}

It is well known that in the early embryo haematopoiesis begins in the blood islands of the yolk sac. Haematopoietic and vascular development is therefore intimately connected, apparently sharing

*Correspondence: Dr G Coukos; E-mail: gcks@mail.med.upenn.edu Received 16 November 2004; revised 27 January 2005; accepted 31 January 2005; published online 22 March 2005 a common mesodermal progenitor, the 'haemangioblast'. Emerging studies have identified a population of primitive endotheliallike cells derived from human embryonic stem cells that includes bipotent cells with endothelial and haematopoietic capacity (Wang et al, 2004). However, until very recently, it was thought that this close association between haematopoiesis and vascularisation was restricted to the developing embryo. Already in 2000, Fernandez Pujol et al (2000) challenged this dogma by showing that CD14 ${ }^{+}$ mononuclear cells cultured in the presence of angiogenic growth factors acquire the markers and morphology of endothelial-like cells. CD14 is expressed by a myeloid population with a remarkable plasticity, since it can comprise monocytes, macrophages, monocyte-derived DCs, monocyte-derived osteoclasts or DC-derived osteoclasts (Shortman and Liu, 2002; Rivollier et al, 2004). Similarly, Schmeisser et al (2001) found that a $>90 \%$ pure population of $\mathrm{CD} 34^{-} \mathrm{CD} 14^{+} \mathrm{CD} 45^{+} \mathrm{CD} 31^{+}$leukocytes expressed endothelial-specific markers after 2 weeks in culture under angiogenic conditions, forming endothelial-like cords and tubular structures in three dimensional Matrigel ${ }^{\circledR}$.

Although the absence of clonal analysis and studies of proliferation cannot exclude that a tinny proportion of proliferating endothelial cells became highly represented in the cultures, these studies suggested for the first time the existence of a novel subset of cells with vasculogenic potential. In addition, a phenotypic overlap between haematopoietic DCs and endothelial cells was soon after described: Fernandez Pujol et al (2001) found that the same population of monocyte-derived DCs could exhibit phenotypic properties of mature DCs under inflammatory conditions, or, alternatively, behave as endothelial-like cells in an angiogenic milieu. The process of 'endothelialisation' was characterised by a disappearance of the leukocyte markers CD14, CD1a and CD83, and the expression of the endothelial markers von Willebrand factor (vWF), vascular endothelial growth factor receptor-2 (VEGFR-2) and VE-cadherin. Although the cells exhibited a reduced capacity to prime T cells, CD86 and major histocompatibility class II (MHC-II) were expressed at similar levels than in mature DCs. Although the purity of the culture is again an issue, monocyte-derived DCs remain in a state of low proliferation, indicating that cultured cells 
have a true mixed EC-dendritic cell phenotype. Another indication that DCs are the closest leukocytes to endothelial cells was provided by Harraz et al. By culturing a population of $\mathrm{CD} 34^{-} \mathrm{CD} 14^{+}$ monocytes under angiogenic conditions, they observed that, in the process of endothelialisation, monocytes acquire a DC phenotype, expressing markers such as endoglin and CDla (Harraz et al, 2001). This in vitro differentiated population could be incorporated into mouse ischemic limbs, although that required coinjection with CD34 ${ }^{+}$cells.

More recently, other studies have confirmed the angiogenic potential of the monocyte/macrophage/dendritic/osteoclast lineage in vitro. By using two generally accepted criteria for the isolation of EPCs, such as uptake of acetylated low-density lipoprotein (LDL) and binding of ulex-lectin, a $\mathrm{CD}_{3}{ }^{-}$population expressing monocyte/macrophage/dendritic markers was isolated from human peripheral blood (Rehman et al, 2003). Although they secreted numerous angiogenic factors, these cells did not exhibit any significant proliferation, suggesting that they may contribute to neovascularisation mainly through migration and incorporation into the vascular wall. A more challenging study has reported pluripotent capabilities for a subset of peripheral blood $\mathrm{CD} 14^{+} \mathrm{CD} 34^{+} \mathrm{CD} 45^{+}$leukocytes. These cells could be isolated and clonally expanded, giving rise not only to endothelial cells under angiogenic conditions but also to lymphocytes, macrophages, epithelial cells, and even liver cells and neurons under appropriate conditions (Zhao et al, 2003).

In an effort to understand our surprising findings in an in vivo model, we have dissected the process of transdifferentiation of murine bone marrow-derived DCs into cells with markers, morphology and functional properties of endothelium. We procured a $>97 \%$ pure population of $\mathrm{CD} 34^{-} \mathrm{CD} 11 \mathrm{c}^{+} \mathrm{DCs}$ by the classical method of culturing bone-marrow cells in the presence of granulocyte-macrophage colony-stimulating factor (GM-CSF). In agreement with their common use as antigenpresenting cells in immunological studies, DCs obtained in this way exhibited all the characteristics of canonical DCs, including dendritic shape, phagocytosis of apoptotic fluorescence-labelled tumour cells and maturation induced by tumour necrosis factoralpha or bacterial lipopolysaccharide in vitro. They also induced antigen-specific T-cell proliferation, as assessed by ${ }^{3} \mathrm{H}$-thymidine incorporation, and secretion of interferon-gamma as well as interleukin-2 by lymphocytes. Interestingly, $\mathrm{CD} 34^{-} \mathrm{CD} 11 \mathrm{c}^{+}$cells cultured in media conditioned by tumour cells expressing high levels of VEGF assumed a spindle-like shape within 3-5 days of culture. After 7 days, $5 \%$ of $\mathrm{CD}^{-} 4^{-} \mathrm{CD} 11 \mathrm{c}^{+}$cells expressed CD31 by immunohistochemistry, while vWF was still not detectable. After 3 weeks, more than $80 \%$ of the cells expressed CD31 and vWF, and expressed binding sites for Griffonia simplicifolia B4 lectin. Incubation of $\mathrm{CD} 34^{-} \mathrm{CD} 11 \mathrm{c}^{+}$immature DCs with tumour cell-conditioned media led to progressive alignment of single cells oriented with the same polarity, forming string-like structures (Figure 1). These grew longitudinally and recruited more cells to the sides, forming cord-like structures. Video integration showed that nearby or distant cells migrated towards alignments or cords of vascular leukocytes (VLCs) adhered to and finally integrated into cord structures.

By flow cytometry, most cells expressed de novo CD34 and upregulated CD31 within 1 week. These changes were not due to overgrowth of a small fraction of contaminating endothelial progenitors, since the rate of proliferation was very low. Instead, we theorised that our analyses captured the progressive 'endothelial-like switch' of $\mathrm{CD} 11 \mathrm{c}^{+}$DCs within the tumour-conditioned media, so we termed these $\mathrm{CD} 11 \mathrm{c}^{+} \mathrm{CD} 45^{+}$DCs expressing CD31 and CD34, and exhibiting morphological and molecular properties of endothelial cells, VLCs. More than $90 \%$ of VLCs exhibited uptake of fluorescent acetylated LDL. Due to the phenotypic overlap between haematopoietic and endothelial cells, electron microscopy is still considered the gold standard to determine the
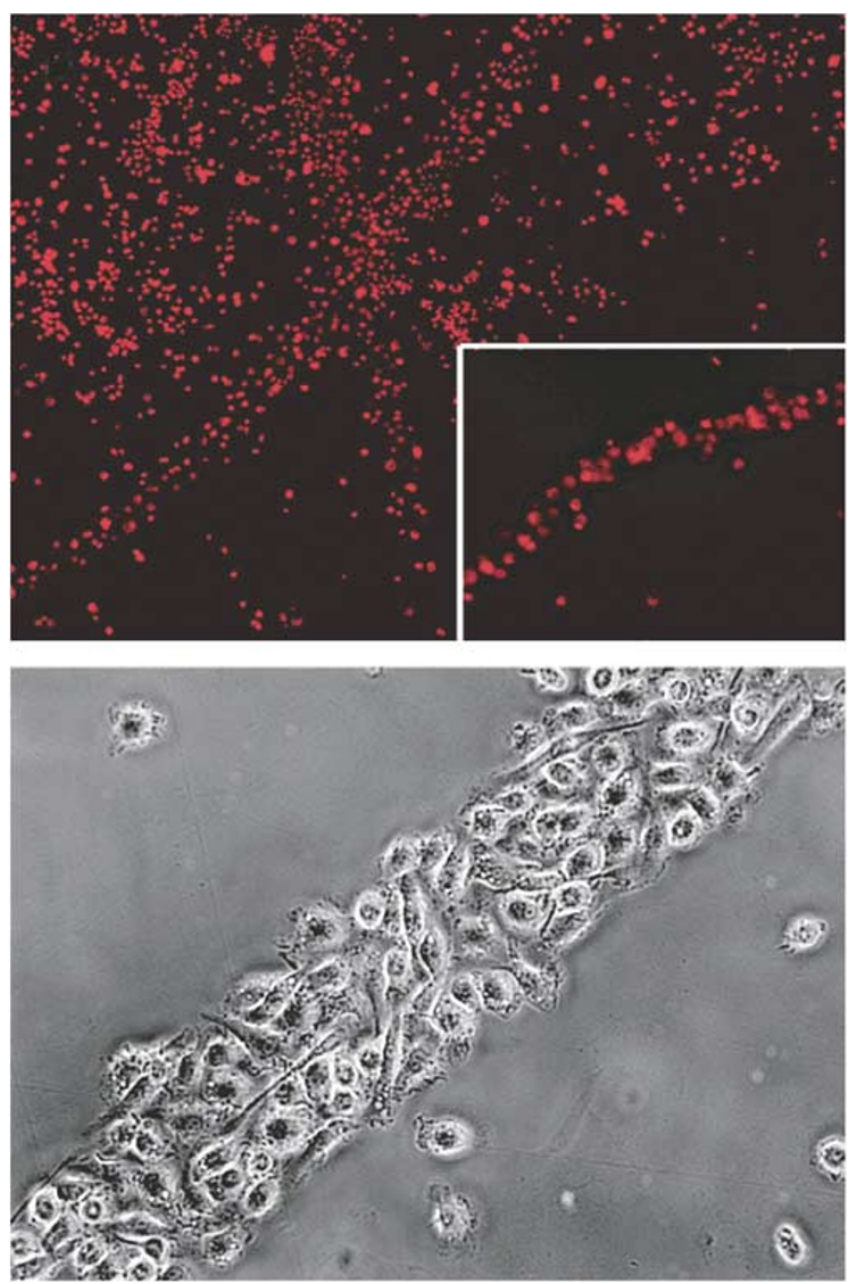

Figure I Transdifferentiation of bone marrow-derived DCs into endothelial-like cells in vitro. Most bone marrow-derived DCs aggregate into cord-like structures after 7 days in culture with tumour cellconditioned media. More than $90 \%$ of them exhibited uptake of fluorescent acetylated low-density lipoprotein (top); Details of a cord composed by bone marrow-derived DCs treated with tumour cell-conditioned media for 2 weeks, which exhibits a cobblestone pattern of cell aggregation (bottom).

nature of a cell. We have showed that within 3 weeks of culture with tumour cell-conditioned media, VLCs exhibited WeibelPalade bodies and endocytic vesicles. In addition, they created intercellular junctions, organising themselves around a lumen, which are typical morphological properties of endothelial cells (Conejo-Garcia et al, 2004).

Remarkably, VEGF, important for the survival and proliferation of bona fide endothelial cells, was the critical factor for endothelialisation. Blocking VEGFR-2 (but not other VEGF receptors) with neutralising antibodies stopped the transdifferentiation process.

\section{VASCULAR LEUKOCYTES CONTRIBUTE TO TUMOUR VASCULARISATION}

Although these in vitro studies revealed an unsuspected plasticity of cells from the monocyte/macrophage/dendritic/osteoclast lineage, it was not clear yet whether these changes took place in vivo, under physiological or pathological conditions. Moreover, although VLCs exhibit ultrastructural properties of endothelial cells, it needed to be proven that they contribute to neovascularisation. 
The inhibitory role of VEGF on the normal differentiation of DC in tumours has been extensively described (Gabrilovich et al, 1996, 1998; Oyama et al, 1998; Ohm et al, 1999): VEGF blocks the functional maturation of DC from haematopoietic progenitor cells by blocking NF- $\kappa \mathrm{B}$ transcription, although it is unlikely that this inhibition alone turns these progenitors into endothelial-like cells. Confirming the critical role of VEGF on tumour immune evasion, antibodies to VEGF enhance the efficacy of cancer immunotherapy by improving endogenous dendritic cell function (Gabrilovich et al, 1999). Furthermore, Almand et al (2000) described that high levels of VEGF in blood result on decreased numbers of competent DCs and accumulation of immature haematopoietic cells. It is tantalising to speculate that these cells might exhibit an endothelial-like phenotype. Nevertheless, the angiogenic potential of DC precursors remained unsuspected until De Palma et al provided evidence that naturally occurring immature myeloid cells could be incorporated into blood vessels in tumours. They transduced bone-marrow progenitors with lentiviral vectors expressing green fluorescent protein under the Tie-2 promoter/enhancer, a marker thought to be endothelial-specific (De Palma et al, 2003). To their surprise, expression of the Tie2p/e vector identified a subset of $\mathrm{CD} 45^{+} \mathrm{CD} 11 \mathrm{~b}^{+} \mathrm{Tie}^{+} \mathrm{Sca}-1^{+} \mathrm{CD} 34^{+}$mononuclear cells that assembled a stromal structure in close association with 'canonical' ECs, a possible involvement in vasculogenesis.

In 2001, we viewed DCs only as critical regulators of adaptive immune responses, including those against tumours. In an attempt to create a model to study the effects of recruiting DCs intratumourally on antitumour immune response, we expressed a newly cloned $\beta$-defensin in an ovarian carcinoma cell line ectopically producing low or high levels of VEGF (Conejo-Garcia et al, 2004). $\beta$ Defensins are antimicrobial peptides that chemoattract immature DCs through CCR6, thus linking innate and adaptive immunity (Yang et al, 2004a). By recruiting DCs to tumour sites, we expected to accelerate phagocytosis and presentation of tumour antigens, thus potentiating the immune response. We indeed found a massive accumulation of $\mathrm{CD} 11 \mathrm{c}^{+}$cells in flank tumours and tumour ascites. $\mathrm{CD} 11 \mathrm{c}$ is a marker mainly restricted to the DC compartment in the mouse. More than $90 \%$ of $\mathrm{CD} 11 \mathrm{c}^{+} \mathrm{CD}_{4} 5^{+}$cells were $\mathrm{MHC}-\mathrm{II}{ }^{+}$, DEC- $205^{+}$and $\mathrm{CD} 8 \alpha^{+}$, documenting their DC lineage, but they expressed very low levels of costimulatory molecules, and were thus identified as DC precursors. In tumours expressing physiologic levels of VEGF (comparable to those found in well vascularised organs such as kidney), expression of $\beta$-defensins reduced tumour growth in a flank model. However, in the presence of increased levels of VEGF, chemoattraction of CCR $6^{+}$DC precursors resulted in a dramatic acceleration of tumour growth and reduced survival compared to tumours with no $\beta$-defensin expression. Although VEGF was ectopically expressed in our model, its levels resemble those found in human tumours. Interestingly, tumours expressing $\beta$ defensins appeared markedly more congested and haemorrhagic compared to controls. They also exhibited a markedly different architecture, with disorganised accumulations of tumour cells, much more abundant $\mathrm{CD}_{3} 1^{+}$vessels and scarce stroma. When we analysed the distribution of $\mathrm{CD} 11 \mathrm{c}^{+} \mathrm{DC}$ precursors by immunohistochemistry, they localised to the luminal surface of capillary-like structures, admixed with $\mathrm{CD} 11 \mathrm{c}^{-}$cells, indicating that they had contributed to the increased vascular density.

The frequency of $\mathrm{CD}_{11 \mathrm{c}^{+}} \mathrm{DC}$ precursors in ascites increased with neoplastic progression, reinforcing the notion that $\mathrm{CD} 11 \mathrm{c}^{+}$ cells may contribute to ovarian carcinogenesis. Similarly to the in vitro experiments, VEGF demonstrated to be a critical factor for the acquisition of morphological and functional characteristics of endothelial cells by DC precursors, since administration of the tyrosine kinase inhibitor SU5416 (Sugen Inc., San Francisco, CA) completely abrogated their vascular homing. Strikingly, we identified for the first time in vivo $\mathrm{CD} 11 \mathrm{c}^{+}$cells exhibiting simultaneous expression of both endothelial and leukocyte markers (VLCs). We hypothesised that our analyses within the tumour microenviron- ment mimicked the progressive 'endothelial-like switch' of CD11c ${ }^{+}$ leukocytes observed in vitro, which appeared to first acquire CD34, increase CD31 expression, and progressively lose the expression of the haematopoietic marker CD45. Tumour-infiltrating VLCs were highly represented in mouse ascites ( $>30 \%$ of total cells). They expressed markers common to endothelial and dendritic cells, such as CD31, vWF mRNA and VEGFR-1 (thought to be specific of endothelial cells); exhibited uptake of acetylated LDL, and bound to Bandeiraea simplicifolia (BS-1) lectin (our unpublished observations). Notably, they also exhibited typical endothelial-specific markers (Rafii and Lyden, 2003), such as VE-cadherin, CD146 (P1H12) and VEGFR-2. More importantly, labelled VLCs transplanted in Matrigel plugs in vivo, assembled into tubular structures formed by fluorescent cells, which were variably perfused with fluorescent dextran injected through the left cardiac ventricle, indicating that they had given rise to intact blood-carrying capillaries in vivo. Additional analysis with fluorochrome-labelled antibodies revealed that cells forming neovessels retain the expression of CD11c and CD45, confirming their leukocyte lineage. Similarly, histological analysis of solid tumour specimens revealed the presence of CD $11 \mathrm{c}^{+}$cells at an endothelial location. Although bona fide endothelial cells can also express CD11c under certain conditions (Langeggen et al, 2002), we also found numerous CD45 ${ }^{+}$ vessels in all the specimens obtained with our tumour model. Of note, the same cells extracted from dissociated tumour specimens or tumour ascites were able to induce strong immune responses in naïve $\mathrm{T}$ cells when treated with a cocktail of inflammatory molecules, confirming their antigen-presenting cell nature.

The accumulation of immunosuppressor, immature $\mathrm{Gr}-1^{+}$cells in mice with large tumour burdens has been already described (Kusmartsev et al, 2000), suggesting that tumours naturally recruit immature myeloid precursors to support their vascularisation. Supporting this idea, we found a population of $\mathrm{CD} 45^{+} \mathrm{CD} 11 \mathrm{c}^{+} \mathrm{VE}-$ cadherin ${ }^{+} \mathrm{CD} 146^{+}$VLCs in a murine lung tumour model, although less represented in the absence of ectopic expression of $\beta$-defensins (unpublished observations). In addition, immature myeloid $\mathrm{Gr}^{+}{ }^{+} \mathrm{CD} 11 \mathrm{~b}^{+}$cells have been recently found to incorporate into tumour endothelium, acquiring endothelial cell properties (Yang et al, 2004b). These cells are more differentiated than classical progenitors, representing immature myeloid cells in an intermediate stage of differentiation. Of note, human tumours of different origins naturally express high levels of $\beta$-defensins, and ovarian cancer in particular produces other chemokines that also attract proangiogenic DCs into the tumour microenvironment, such as stroma-derived factor 1 (SDF-1) (Curiel et al, 2004). Therefore, it was not surprising to find that two different antiCD45 antibodies stained tortuous, thorny vascular structures that were simultaneously positive for vascular markers in human ovarian carcinoma specimens exhibiting high levels of VEGF and $\beta$-defensins mRNA.

The concept that the formation of blood vessels by DCs with endothelial potential (VLCs) is not restricted to artificial tumour models, but rather constitutes a bona fide angiogenic mechanism promoting the progression of human tumours, is supported by the high $(\sim 40 \%)$ proportion of VLCs systematically accumulated in ovarian carcinomas (Conejo-Garcia et al, 2005). Single-cell suspensions derived from freshly dispersed human ovarian carcinomas separate into three clearly isolatable fractions, according to their expression of CD45 (leukocyte) and VE-cadherin (endothelial) markers: bona fide leukocytes (CD45 ${ }^{+}$VE-cadherin ${ }^{-}$; mostly lymphocytes according to their FSC vs SSC profile), bona fide endothelial cells (CD45 ${ }^{-}$VE-cadherin $\left.{ }^{+}\right)$and, VLCs $\left(\mathrm{CD} 45^{+}\right.$VE-cadherin $\left.{ }^{+}\right)$. We have identified these three populations in all specimens analysed so far $(n=14)$, though in different proportions. Although the high frequency of VLCs in solid tumours may be caused by the mechanical procedure of dispersion, it is still surprising that the proportion of VLCs is comparable to that of non-VLCs. 
In addition to VE-cadherin, VLCs express other endothelial markers, such as CD146 and CD34. Further characterisation of VLCs revealed that $>90 \%$ are ${\mathrm{MHC}-\mathrm{II}^{+}}^{+}$and $\mathrm{CD} 86^{+}$, while $>86 \%$ express $\mathrm{CD}_{11 \mathrm{c}^{+}}$, compatible with an immature DC phenotype. Supporting their immature character, $45 \%$ of VLCs express $\mathrm{CD} 133^{+}(15-80 \%)$, a stem-cell marker with unknown function, absent in mature endothelium or committed myeloid cells. Confirming their angiogenic potential, tumour-infiltrating VLCs have the capacity to create perfusible blood vessels in vivo in Matrigel plugs. Experiments performed with CCR6-immunotoxins suggest that the role of VLCs to tumour vasculogenesis takes place primarily during the early stages of tumour growth. Due to their limited proliferating capacity, we propose that their angiogenic potential is mediated mainly by their recruitment to the tumour.

In addition to their capacity to become endothelial-like cells, a recent study reported the presence of $\mathrm{CD}_{11 \mathrm{~b}}{ }^{+}$leukocytes as periendothelial vascular mural cells (Rajantie et al, 2004). We have also found $\mathrm{CD}_{11 \mathrm{c}^{+}}$at a periendothelial location in selected specimens of our tumour model, although they always expressed CD31, a marker typically absent in classical pericytes. It is likely that these periendothelial leukocytes contribute to neovascularisation by secreting angiogenic factors. Alternatively, they may be VLCs assembling into neovessels branching out, or represent leukocytes transdifferentiating into pericytes.

\section{VLCS REPRESENT A NOVEL TARGET FOR IMMUNOTHERAPY}

The potential to inhibit tumour growth by targeting VLCs is illustrated by our studies with CCR6-immunotoxins: Tumours injected in the presence of anti-CCR6 antibodies bound to saporin, a ribosomal-inactivating protein, grew 2.6-fold less than tumours injected with an isotype control antibody. Since decrease in tumour growth was associated with a reduced number of $\mathrm{CD} 11 \mathrm{c}^{+}$infiltrating cells, VLCs emerge as novel therapeutic targets. Antibodies directed against specific antigens and conjugated to toxins may contribute to block tumour vascularisation. On the other hand, we have found a higher presence of VLCs in the tumour periphery, suggesting that, as the tumour grows, VLCs stick preferentially around the tumour. Therefore, VLCs appear as optimal candidates for targeted delivery of therapeutic agents as 'Trojan Horses'. For instance, adoptive therapy of VLCs expressing suicidal genes with bystander effect may help to collapse tumour neovessels, thus triggering both necrosis and antitumour immune responses. Alternatively, transduction of VLCs with vectors expressing immune factors under the control of endothelial-specific promoters may negate their angiogenic potential once they have been incorporated into the vessel wall, delivering inflammatory cytokines into the tumour microenvironment.

\section{TRANSDIFFERENTIATION IN THE TUMOUR MICROENVIRONMENT OR RECRUITMENT AS DUAL CELLS?}

An important question that remains unresolved is whether VLCs are directly recruited as cells with 'haemangioblast' properties, or rather as leukocytes in a low stage of differentiation that turn into dual cells in the tumour microenvironment (Figure 2). Several studies conducted by our group support the later hypothesis: First, we have not found $\mathrm{CD} 45^{+} \mathrm{CD} 11 \mathrm{c}^{+}$VE-cadherin ${ }^{+} \mathrm{CD} 146^{+}$cells in peripheral blood of patients with ovarian carcinoma. Similarly, we

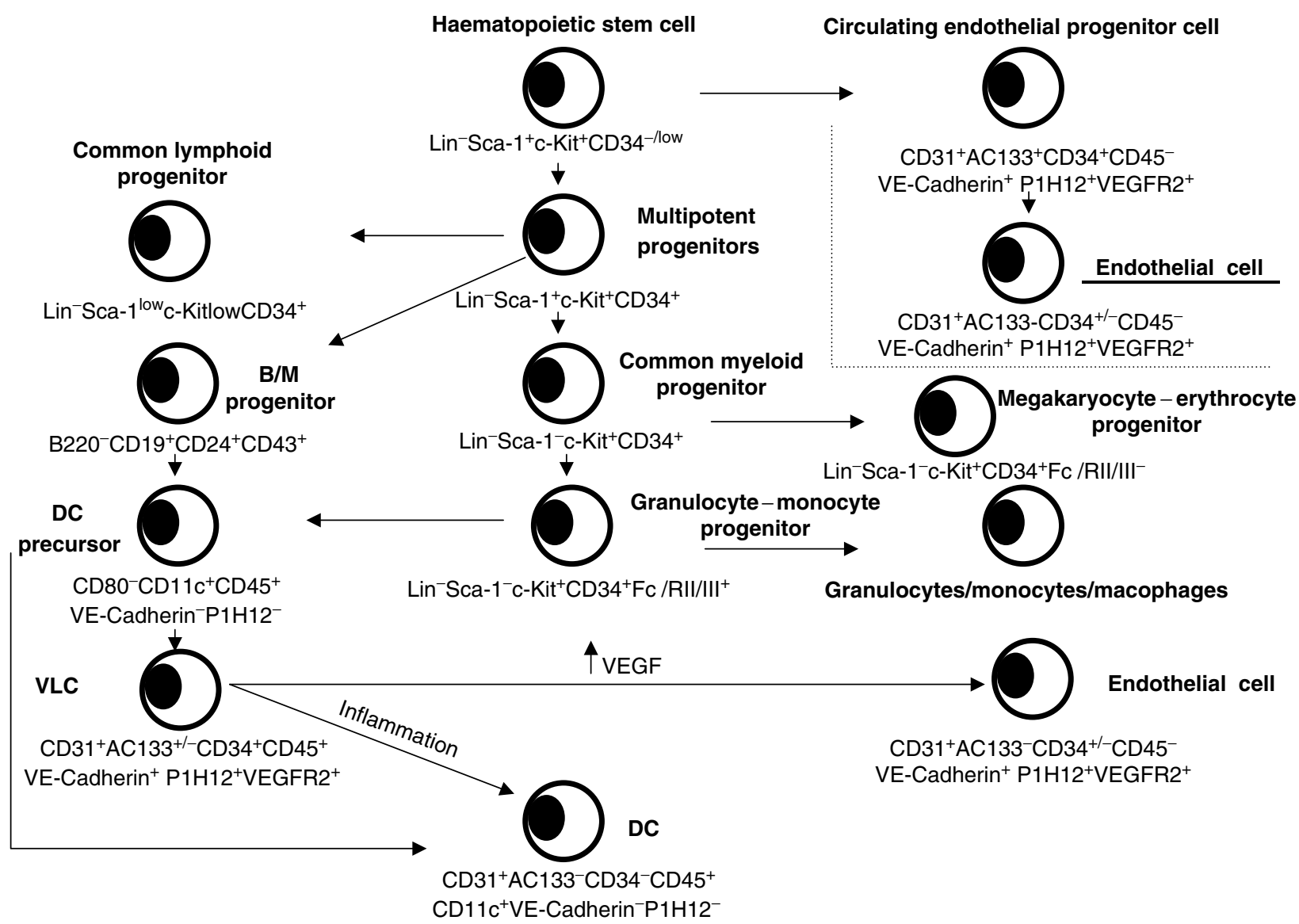

Figure 2 Hypothetical scheme of the transdifferentiation of DC precursors into endothelial-like vascular leukocytes (VLCs) in mouse tumours. DC: dendritic cells; $M \Phi$ : macrophage. 
have detected VLCs in different bone-marrow and lymphoid organs of tumour-bearing mice at very low frequencies $(<0.2 \%)$. Second, we have demonstrated that the tumour microenvironment induces DC to differentiate in vivo into endothelial-like cells. We injected BM-derived DCs labelled with a fluorochrome subcutaneously around tumours overexpressing VEGF. At 3 days postinjection, fluorescent DCs microdissected by laser-capture exhibited a 2.6- and 6.1-fold upregulation of CD31 and VEcadherin, respectively, compared to preinjection levels, while 7 days postinjection a 60 - and 22-fold upregulation was noted, respectively, by real-time quantitative PCR. Furthermore, VEGFR2 was upregulated 20 fold. Collectively, these data suggest that the tumour microenvironment can induce the endothelialisation of immature DCs, thus mimicking the transdifferentiation process observed in vitro. In addition, we have observed that labelled bone marrow-derived DCs assemble into neovessels in Matrigel plugs, a process that can be blocked by addition of neutralising antibodies against VEGFR-2. However, although they are generally used for every kind of immunological studies, it is still possible that bone marrow-derived DCs do not belong to the DC type naturally found in tumours and possess more plasticity.

\section{CONCLUDING REMARKS}

The identification of DCs or DC precursors as bipotent cells has important implications for the physiopathology and therapy of tumours. As VLCs are an important host cell population affecting tumour growth, important concerns emerge regarding the use of intratumoural DC inoculation as a means for tumour vaccination in the human. Similarly, if VLCs are inefficient APCs, what is their exact role in immune tolerance of tumours? It is also intriguing to hypothesise that DC precursors might turn into lymphatic endothelial-like cells under the influence of VEGF-C, thus contributing to tumour metastasis. Finally, important questions arise on numerous issues. Which is the relative contribution of VLCs to tumour vascularisation? At which point of tumour development is it more relevant? Is the contribution of VLCs to vasculogenesis organ-dependent or affects different tumours in similar proportions? And most importantly, are VLCs contributing to physiologic vascularisation? The existing evidence that endothelium increases APC capabilities and specialised endothelium may participate in maintenance of peripheral tolerance raises intriguing questions on the ontology of endothelial cells in normal tissues and the relationship of angiogenesis and peripheral tolerance.

\section{ACKNOWLEDGEMENTS}

This work was supported by NCI ovarian SPORE P01-CA83638, NIH R01 CA098951 and by the Sidney Kimmel Foundation. FB was supported by NIH Research Grant D43-TW00671 funded by the Fogarty International Center. RJB was supported by NIH/NICHD K12-HD43459 Career Development in Women's Health Research.

\section{REFERENCES}

Almand B, Resser JR, Lindman B, Nadaf S, Clark JI, Kwon ED, Carbone DP, Gabrilovich DI (2000) Clinical significance of defective dendritic cell differentiation in cancer. Clin Cancer Res 6: 1755-1766

Asahara T, Murohara T, Sullivan A, Silver M, van der Zee R, Li T, Witzenbichler B, Schatteman G, Isner JM (1997) Isolation of putative progenitor endothelial cells for angiogenesis. Science 275: 964-967

Conejo-Garcia JR, Benencia F, Courreges MC, Kang E, Mohamed-Hadley A, Buckanovich RJ, Holtz DO, Jenkins A, Na H, Zhang L, Wagner DS, Katsaros D, Caroll R, Coukos G (2004) Tumor-infiltrating dendritic cell precursors recruited by a beta-defensin contribute to vasculogenesis under the influence of Vegf-A. Nat Med 10: 950-958

Conejo-Garcia JR, Buckanovich RJ, Benencia F, Courreges MC, Rubin SC, Carroll RG, Coukos G (2005) Vascular leukocytes contribute to tumor vascularization. Blood 105: 679-681

Curiel TJ, Cheng P, Mottram P, Alvarez X, Moons L, Evdemon-Hogan M, Wei S, Zou L, Kryczek I, Hoyle G, Lackner A, Carmeliet P, Zou W (2004) Dendritic cell subsets differentially regulate angiogenesis in human ovarian cancer. Cancer Res 64: 5535-5538

De Palma M, Venneri MA, Roca C, Naldini L (2003) Targeting exogenous genes to tumor angiogenesis by transplantation of genetically modified hematopoietic stem cells. Nat Med 9: 789-795

Fernandez Pujol B, Lucibello FC, Gehling UM, Lindemann K, Weidner N, Zuzarte ML, Adamkiewicz J, Elsasser HP, Muller R, Havemann K (2000) Endothelial-like cells derived from human CD14 positive monocytes. Differentiation 65: $287-300$

Fernandez Pujol B, Lucibello FC, Zuzarte M, Lutjens P, Muller R, Havemann K (2001) Dendritic cells derived from peripheral monocytes express endothelial markers and in the presence of angiogenic growth factors differentiate into endothelial-like cells. Eur J Cell Biol 80: $99-110$

Folkman J (2002) Looking for a good endothelial address. Cancer Cell 1: $113-115$

Gabrilovich D, Ishida T, Oyama T, Ran S, Kravtsov V, Nadaf S, Carbone DP (1998) Vascular endothelial growth factor inhibits the development of dendritic cells and dramatically affects the differentiation of multiple hematopoietic lineages in vivo. Blood 92: 4150-4166

Gabrilovich DI, Chen HL, Girgis KR, Cunningham HT, Meny GM, Nadaf S, Kavanaugh D, Carbone DP (1996) Production of vascular endothelial growth factor by human tumors inhibits the functional maturation of dendritic cells. Nat Med 2: 1096-1103
Gabrilovich DI, Ishida T, Nadaf S, Ohm JE, Carbone DP (1999) Antibodies to vascular endothelial growth factor enhance the efficacy of cancer immunotherapy by improving endogenous dendritic cell function. Clin Cancer Res 5: 2963-2970

Harraz M, Jiao C, Hanlon HD, Hartley RS, Schatteman GC (2001) CD34blood-derived human endothelial cell progenitors. Stem Cells 19: 304-312

Kalka C, Masuda H, Takahashi T, Kalka-Moll WM, Silver M, Kearney M, Li $\mathrm{T}$, Isner JM, Asahara T (2000) Transplantation of ex vivo expanded endothelial progenitor cells for therapeutic neovascularization. Proc Nat Acad Sci USA 97: 3422-3427

Kusmartsev SA, Li Y, Chen SH (2000) Gr-1+ myeloid cells derived from tumor-bearing mice inhibit primary $\mathrm{T}$ cell activation induced through CD3/CD28 costimulation. J Immunol 165: 779-785

Langeggen H, Berge KE, Johnson E, Hetland G (2002) Human umbilical vein endothelial cells express complement receptor 1 (CD35) and complement receptor $4(\mathrm{CD} 11 \mathrm{c} / \mathrm{CD} 18)$ in vitro. Inflammation 26: $103-110$

Ohm JE, Shurin MR, Esche C, Lotze MT, Carbone DP, Gabrilovich DI (1999) Effect of vascular endothelial growth factor and FLT3 ligand on dendritic cell generation in vivo. J Immunol 163: $3260-3268$

Oyama T, Ran S, Ishida T, Nadaf S, Kerr L, Carbone DP, Gabrilovich DI (1998) Vascular endothelial growth factor affects dendritic cell maturation through the inhibition of nuclear factor-kappa B activation in hemopoietic progenitor cells. J Immunol 160: 1224-1232

Rafii S (2000) Circulating endothelial precursors: mystery, reality, and promise. J Clin Invest 105: 17-19

Rafii S, Lyden D (2003) Therapeutic stem and progenitor cell transplantation for organ vascularization and regeneration. Nat Med 9: 702-712

Rajantie I, Ilmonen M, Alminaite A, Ozerdem U, Alitalo K, Salven P (2004) Adult bone marrow-derived cells recruited during angiogenesis comprise precursors for periendothelial vascular mural cells. Blood 104: $2084-2086$

Rehman J, Li J, Orschell CM, March KL (2003) Peripheral blood 'endothelial progenitor cells' are derived from monocyte/macrophages and secrete angiogenic growth factors. Circulation 107: 1164-1169

Reyes M, Dudek A, Jahagirdar B, Koodie L, Marker PH, Verfaillie CM (2002) Origin of endothelial progenitors in human postnatal bone marrow. J Clin Invest 109: 337 - 346 
Rivollier A, Mazzorana M, Tebib J, Piperno M, Aitsiselmi T, RabourdinCombe C, Jurdic P, Servet-Delprat C (2004) Immature dendritic cell transdifferentiation into osteoclasts: a novel pathway sustained by the rheumatoid arthritis microenvironment. Blood 104: 4029-4037

Ruoslahti E (2002) Specialization of tumour vasculature. Nat Rev Cancer 2: $83-90$

Schmeisser A, Garlichs CD, Zhang H, Eskafi S, Graffy C, Ludwig J, Strasser RH, Daniel WG (2001) Monocytes coexpress endothelial and macrophagocytic lineage markers and form cord-like structures in Matrigel under angiogenic conditions. Cardiovasc Res 49: 671-680

Shortman K, Liu YJ (2002) Mouse and human dendritic cell subtypes. Nat Rev Immunol 2: 151 - 161

Wang L, Li L, Shojaei F, Levac K, Cerdan C, Menendez P, Martin T, Rouleau A, Bhatia M (2004) Endothelial and hematopoietic cell fate of human embryonic stem cells originates from primitive endothelium with hemangioblastic properties. Immunity 21: 31-41

Yang D, Biragyn A, Hoover DM, Lubkowski J, Oppenheim JJ (2004a) Multiple roles of antimicrobial defensins, cathelicidins, and eosinophil-derived neurotoxin in host defense. Annu Rev Immunol 22: $181-215$

Yang L, Debusk LM, Fukuda K, Fingleton B, Green-Jarvis B, Shyr Y, Matrisian LM, Carbone DP, Lin PC (2004b) Expansion of myeloid immune suppressor $\mathrm{Gr}+\mathrm{CD} 11 \mathrm{~b}+$ cells in tumor-bearing host directly promotes tumor angiogenesis. Cancer Cell 6: $409-421$

Zhao Y, Glesne D, Huberman E (2003) A human peripheral blood monocyte-derived subset acts as pluripotent stem cells. Proc Natl Acad Sci USA 100: $2426-2431$ 